\title{
CLA-MAC: A Cooperative Extension of Load Adaptive MAC Protocol
}

\author{
Howard Huang Homayoun Yousefi'zadeh Hamid Jafarkhani \\ Center for Pervasive Communications and Computing \\ University of California, Irvine \\ [hthuang, hyousefi, hamidj] @uci.edu
}

\begin{abstract}
The use of cooperative communications techniques has proven to significantly improve network performance. In this paper, we propose a cooperative extension of load adaptive MAC (LA-MAC) protocol proposed earlier by us as a hybrid CSMA-TDMA protocol for MANETs. We describe the design and GNU radio implementation of our protocol to which we refer as cooperative LA-MAC (CLA-MAC). Our protocol design takes into account frame structure, message exchange, multiple relay selection policies, and contention avoidance. The relay selection policies of the protocol include two practically viable alternatives, namely, the nearest neighbor and largest harmonic mean channel. Experiments confirm that both relay selection policies proposed by CLA-MAC protocol can achieve lower packet error rates and smaller packet delays compared to the original LA-MAC in the cases of poor signal quality reception at a destination.
\end{abstract}

\section{INTRODUCTION}

In the recent few years, several cooperative communication medium access control (MAC) protocols have been proposed and implemented in real wireless systems [2], [10], [12]. The authors of [16] propose a cooperative protocol named CoopMAC for IEEE 802.11 networks. The authors of [21] propose a distributed coordination function (DCF) based MAC layer protocol, called $\mathrm{rDCF}$, to enhance the multi-rate relaying performance. Another DCF based cooperative MAC protocol is automatic relay cooperative MAC (ARC-MAC) [14]. In [20], a cooperative MAC scheme was implemented based on the wireless open-access research platform (WARP) platform, a software defined radio (SDR) developed by Rice university.

In this paper, we propose a cooperative extension of load adaptive MAC (LA-MAC) [8] protocol proposed earlier by us as a hybrid CSMA-TDMA [11] protocol for MANETs. We describe the design and GNU Radio [4] implementation of our protocol to which we refer as cooperative LA-MAC (CLA-MAC). We host the GNU Radio implementation of our protocol in universal software radio peripheral (USRP) [7], [6] the SDR platform of choice used in our lab. To the best of our knowledge, this is the first cooperative MAC protocol designed for and implemented in GNU Radio.

In our protocol, each node listens and stores packets broadcast by other nodes, even if it is not the intended destination. The destination chooses the nodes with the best channel as a relay and the relay forwards the decoded-and-reencoded data to the destination. We utilize a pair of distributed relay selection policies namely the nearest neighbor and the largest harmonic mean channel. The latter has its roots in the opportunistic relaying work of [3] and functions based on selecting

This work was supported in part by a research contract from the Boeing Company. the best instantaneous channel conditions. Experimental results obtained from our USRP based MANET testbed show that the packet error rate (PER), bit error rate (BER), and packet delay can be significantly reduced in many scenarios of interest.

The rest of this paper is organized as follows. In Section II, the system overview including GNU Radio and LA-MAC is introduced. The proposed cooperative MAC protocol is described in Section III. The implementation issues and experimental results are discussed in Section IV. Conclusions are drawn and future work is summarized in Section V.

\section{SySTEM OVERVIEW}

In this work, our goal is to design a cooperative extension of LA-MAC protocol and implement it in USRP units running on GNU Radio. In the following two subsections, we provide a brief overview of the building blocks used for the design and implementation of our proposed protocol.

\section{A. USRP-Based MANET Nodes}

In our earlier work of [15], we described the details of the formation of our MANET testbed using a collection of SDR nodes. Each node consists of a USRP unit connected to a general purpose processing (GPP) host PC running a Linux operating system.

The USRP motherboard contains a field-programmable gate array (FPGA), analog-to-digital converters (ADC), digital-toanalog converters (DAC), and a number of slots for connecting various RF front-end daughterboards that provide TX and RX functionality. The main use of the motherboard is to provide baseband processing. The USRP is connected to the host PC through a high-speed USB2 interface.

\section{B. PHY Implementation}

We use GNU Radio software package to program a MIMOcapable PHY layer (as well as the MAC protocol) within USRP units. Our PHY implementation utilizes a customized MIMO-OFDM PHY that works based an open-source implementation of IEEE 802.11n described in [22]. The open-source library supports many features of 802.11n PHY, including space-time block coding and channel estimation. Our implementation of both PHY and MAC layers utilize the m-block framework [23] instead of the signal processing blocks and flow graph framework found in standard GNU Radio. 


\section{LA-MAC Protocol}

In this subsection, we describe LA-MAC protocol the building block of CLA-MAC protocol proposed in this paper. The basic idea of LA-MAC is to adaptively switch among three different modes of operation, namely, carrier sense multiple access (CSMA), time-division multiple access (TDMA), and a "HYBRID" mode according to network traffic load. To reduce overhead, none of the three modes of LA-MAC uses readyto-send (RTS) and clear-to-send (CTS) messages. In its main mode of operation, each node is assigned its own time slot to transmit but can also compete for transmission in other time slots. Each node sends a list of its one-hop neighbors to other nodes, so after the initialization phase of the protocol each node has its two-hop neighborhood information. The protocol starts operating in the CSMA mode at the initialization phase and implicitly switches to the Hybrid mode as its main mode of operation past that phase. Operation in the TDMA mode can be forced by disabling the competition algorithm of the protocol in the Hybrid mode. There are two statues of operation in the Hybrid mode for each node: low contention (LC) and high contention (HC). The protocol switches from one statue to another based on two collision thresholds. In the LC statue, nodes can compete for any time slot belonging to its two-hop neighbors while in the HC statue, a node can only complete for its one-hop neighbors' time slot. Each node has a higher priority than the other nodes in its own slot. LAMAC minimizes the probability of facing the hidden terminal problem through the use of its competition algorithm. LAMAC relies on designated nodes transmitting SYN messages within their two-hop neighborhoods to allow time slot synchronization among the nodes of each neighborhood.

\section{Cooperative LA-MAC (CLA-MAC) Protocol}

In this section, we present our proposed CLA-MAC protocol. We note that CLA-MAC preserves all of the advantages of LA-MAC in terms of its operating mechanism and performance advantages. Consistent with the design of LAMAC and in order to reduce the signaling overhead, RTS and CTS signals are not used in CLA-MAC. However, CLAMAC provides an improved alternative of message delivery through relaying messages over time-varying topologies where the quality of links may deteriorate for a period of time over which the transmission may get performed. In this paper, we consider the following scenario for implementing cooperative communications. A source transmits its packets to a destination. During the source's transmission, all nodes in a relay candidate pool listen and store the source packet they receive. After the source finishes its transmission, the destination will decide if a relay is needed and which node should be selected as the relay. In particular, if the direct source-destination link is weak and the destination fails to decode a packet transmitted from the source, one node from the candidate pool will be selected as a cooperative relay. Fig. 1 shows the message exchange among a Src, relay, and Dst. In the figure, Src transmits data to Dst. Two relays (Relay 1 and Relay 2) as well as Dst receive the data. Since Dst cannot decode the message correctly, it sends a relay request

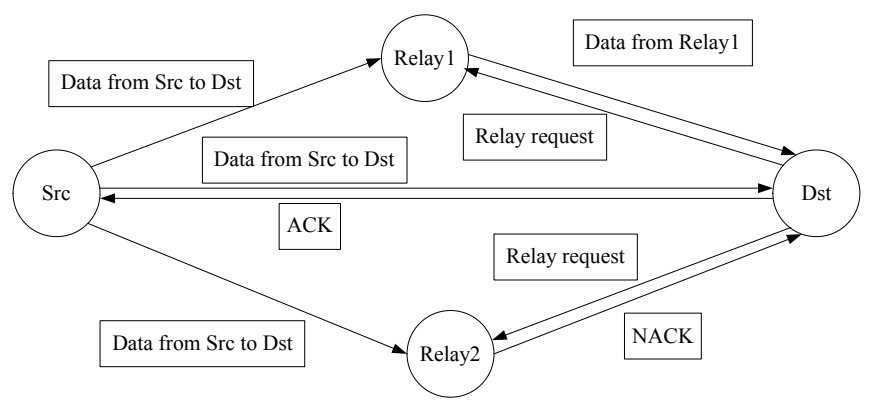

Fig. 1. Message exchange among source, destination, and relays.

to the node with the best channel condition, Relay 2, in its relay candidate pool. Since Relay 2 cannot decode the packet either, it sends back a NACK the destination. Then, Dst tries the second best node, Relay 1, and receives the correct data from Relay 1.

\section{A. Frame Format}

The frame structure of the protocol is illustrated in Fig. 2. In a frame, three bits are used to define the frame type. In Fig. 2, Src and Dst represent the MAC addresses of sender and receiver; Relay is the relay address if required in the transmission; Chan stores the channel quality information between the sender and the receiver. In the data field, the maximal data length is currently configured to 2048 bytes. The message types are defined in Fig. 3.

It is important to note that in Fig. 2, Type is a single bit flag specifying whether a frame is a control or data frame. The two SubType bits define the details of a frame. The combination of Type and SubType bits cover all possible frame types used by CLA-MAC.

\begin{tabular}{|c|c|c|c|c|c|}
\hline Type & SubType & Src & Dst & Relay & Data \\
\hline
\end{tabular}

Fig. 2. An illustration of the frame format in CLA-MAC protocol.

\begin{tabular}{|c|c|c|}
\hline Type & SubType & Description \\
\hline 0 & 00 & Control frame, ACK \\
\hline 0 & 01 & Control frame, NACK \\
\hline 0 & 10 & Control frame, Relay request \\
\hline 0 & 11 & Control frame, new node broadcast \\
\hline 1 & 00 & Data frame, from Src to Dst \\
\hline 1 & 01 & Data frame, from relay to Dst \\
\hline 1 & 10 & $\begin{array}{l}\text { Data frame, for the new node to } \\
\text { broadcast table }\end{array}$ \\
\hline 1 & 11 & $\begin{array}{l}\text { Data frame, for other nodes to re- } \\
\text { ply to the new node }\end{array}$ \\
\hline
\end{tabular}

Fig. 3. The definition of message types in CLA-MAC protocol. 


\section{B. Initialization Phase}

The initialization phase of CLA-MAC builds on that of LAMAC, i.e., each nodes obtains information about its twohop neighbors.

Each node maintains a table describing the MAC address, timestamp, and channel quality associated with each node in a one-hop neighborhood. For each entry in the table there is another table of that node's one-hop neighbors. While the inclusion of MAC address and timestamps is common to LAMAC and CLA-MAC, the inclusion of channel quality is specific to CLA-MAC. For both protocols, the MAC address entry stored in the table is included in the frame received from another node and the timestamp is generated by a local clock.

When a new node joins the network, it collects information on its neighbors using a broadcast frame that includes its MAC address. The first two fields of the frame are set to 011. The one-hop neighbors use the broadcast to determine the channel quality of the new node, and add the information to their own tables. Then, every node sends a frame with its own MAC address and table to the new node. The first two fields of the frame are set to 111 . Finally, the new node broadcasts its newly constructed table to all of its one-hop neighbors. The first two fields of the frame are set to 110 . The initialization process repeats periodically to detect changes in network topology. The initialization phase is illustrated in Fig. 4.

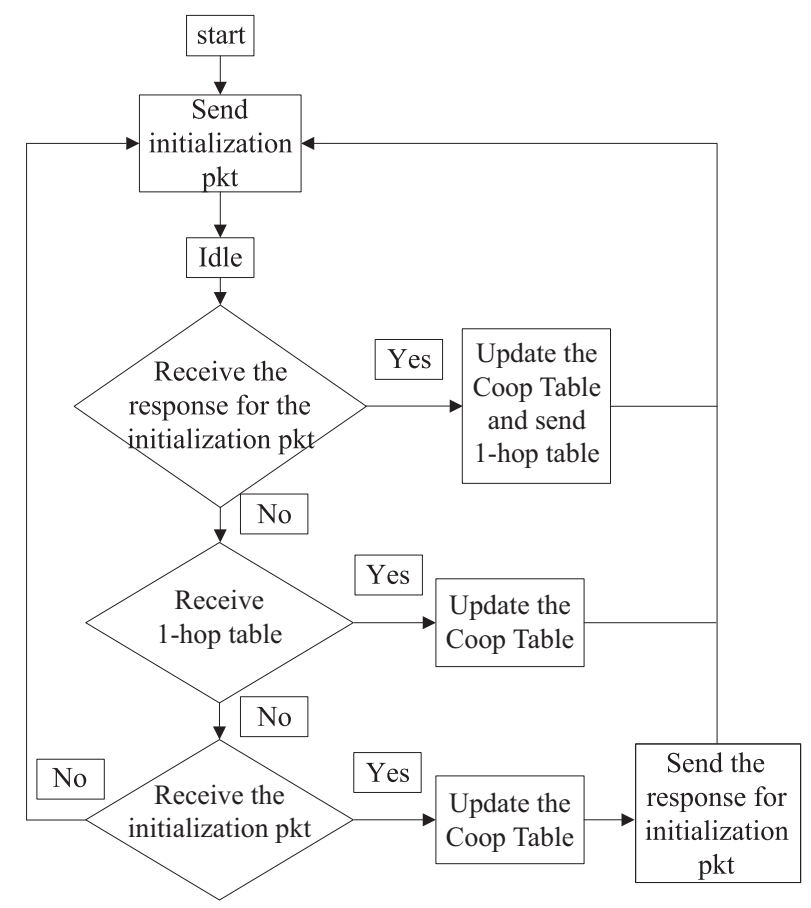

Fig. 4. The flow chart of the initialization phase of CLA-MAC protocol.

\section{Source-to-Destination Transmission}

At a given time slot, the node that is given the right to transmit data is a source, and all other one-hop neighborhood nodes except possibly the destination become relay candidates. The source sends a frame with "100" in the first two fields. If the destination can decode the source data correctly (i.e., the
CRC check is passed), it sends back an ACK message and the transmission is completed.

The timestamp generated by LA-MAC for synchronization purposes is also used by CLA-MAC. During the source's transmission, all nodes in the relay candidate pool listen and store the received source packet. Further, each node in the relay candidate pool sets a timestamp upon the arrival of that packet. If the timestamp expires and a node does not receive a relay request, it discards the source packet.

\section{Decode-and-Forward Based Relaying}

When using decode-and-forward strategy, the relay decodes a packet received from the source, re-encodes it, and then sends it to the destination. The first two fields of the relay's frame are "101". The destination can combine the received packets from both the source and relay for decoding. When the relay-destination channel is much better than the sourcedestination channel, the destination could simply use the received packet from the relay for decoding. The destination will send back an ACK to the source if it decodes the packet data correctly. Cyclic redundancy check (CRC) is used to check whether the received packet is successfully decoded, and this information is then passed to the MAC layer through the use of m-block messages.

\section{E. Relay Selection}

If the destination fails to decode the source data, it will send a relay request. The Dst field of the frame is the MAC address of the selected relay. The destination will search its table and selects the candidate with best channel quality. If the selected relay has decoded the source data correctly, it will then start to send the data to the destination in the subsequent slot.

Otherwise and if the selected relay cannot decode the source data either, it will send a NACK message to the destination indicating that it declines to become the relay. Then the destination will select another relay according to the channel quality criterion from the remaining candidates. It is important to note that it may not be necessary to consider all nodes of the candidate pool since the probability of a successful decoding is small for a poor link quality. In practice, only the nodes whose channel qualities are better than a pre-defined threshold are considered as candidates. This process repeats until a feasible relay is found, or all candidates cannot serve as a relay. If the destination does not receive a packet after a pre-defined time threshold, it assumes that no relay is available. The processes of cooperative transmission in relay and destination are illustrated in Fig. 5 and Fig. 6, respectively.

In [3], the authors define two abstract policies for selecting the relay that can provide the best end-to-end channel between a source and destination pair. The first policy selects a relay with the largest minimum of the two source-relay and relaydestination channels, while the second policy selects a relay with the largest harmonic mean of the channels. Based on the second absract policy of [3], CLA-MAC designs and implements a channel quality metric using signal-to-noiseratio (SNR). Since CLA-MAC does not utilize RTS/CTS handshakes for collision avoidance unlike what is proposed 


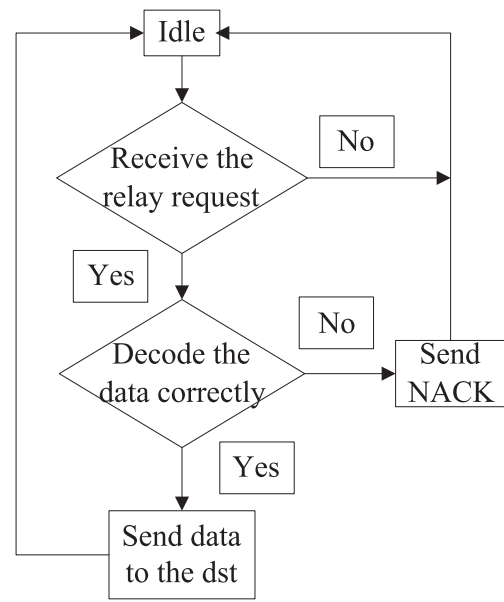

Fig. 5. A flow chart of relay operation.

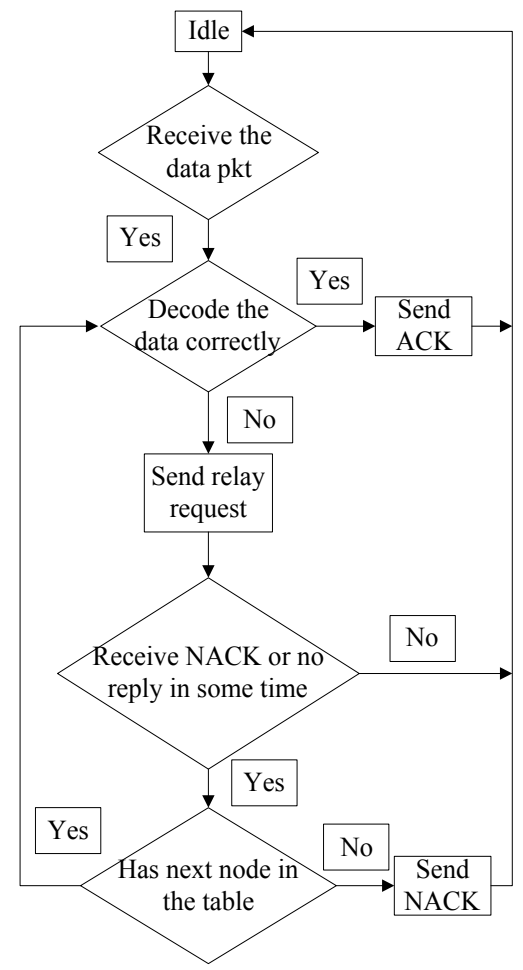

Fig. 6. A flow chart of destination operation.

in the policy of [3], it estimates the two channels using packets sent during the initialization phase. No extra overhead is incurred to provide the destination with the channel information, since the three-way handshake used to acquire the two-hop neighborhood is handled by LA-MAC. CLAMAC then simply piggybacks the channel information on the initialization process. The channel metric policy is expressed as

$$
h_{i}=\frac{2}{\frac{1}{\operatorname{SNR}_{s, i}}+\frac{1}{\operatorname{SNR}_{i, d}}}
$$

where $\mathrm{SNR}_{s, i}$ and $\mathrm{SNR}_{i, d}$ are the SNR of source-relay and relay-destination in the case of $i^{\text {th }}$ relay, respectively. Accord- ing to this policy, the relay that maximizes the function $h_{i}$ is selected as the best relay.

As a second policy, CLA-MAC also supports the "nearest neighbor" selection policy in which the relay offering the highest SNR to the destination is chosen.

\section{F. Analysis}

In this subsection, we provide an analytical insight as to how the use of our cooperative MAC protocol can improve performance. For many practical modulation types such as phase shift keying (PSK) and quadrature amplitude modulation (QAM) with coherent demodulation, it is well known that the bit error probability in an additive white Gaussian noise (AWGN) channel can be approximated as

$$
P_{b}(\gamma)=\alpha Q(\sqrt{\beta \gamma})
$$

where $\alpha$ and $\beta$ are constants related to specific modulation types, $\gamma$ is the per symbol SNR, and the function $Q$ is the tail probability of the standard Gaussian distribution.

Under the widely used Rayleigh channel, the SNR is exponentially distributed and its probability density function is given by $f_{\gamma}(\gamma)=(1 / \bar{\gamma}) \exp \{\gamma / \bar{\gamma}\}$, where $\bar{\gamma}$ is the average per symbol SNR. The average bit error probability is obtained as

$$
\begin{aligned}
\bar{P}_{b}(\bar{\gamma}) & =E_{\gamma}\{\alpha Q(\sqrt{\beta \gamma})\} \\
& =\frac{\alpha}{2}\left[1-\sqrt{\frac{\beta \bar{\gamma}}{2+\beta \bar{\gamma}}}\right] \approx \frac{\alpha}{2 \beta \bar{\gamma}}
\end{aligned}
$$

where the approximation is valid for a high SNR. In our work, we utilize the 16-QAM modulation using Gray coding to map the bits into the symbols. For the selections of our work, the typical values of the parameters are $\alpha=0.75$ and $\beta=0.2$ [18].

When the transmitted symbols are sufficiently interleaved, we may consider that individual symbols within a packet are independently affected by fading. Suppose that the channel decoder at a receiver can correct up to $k$ bit errors for a packet with $N$ information bits. Then, the PER is expressed as

$$
P_{p}(\bar{\gamma})=1-\sum_{i=0}^{k}\left(\begin{array}{c}
N \\
i
\end{array}\right)\left(\bar{P}_{b}\right)^{i}\left(1-\bar{P}_{b}\right)^{N-i}
$$

The bit or frame error probability is a decreasing function of the average SNR $(\bar{\gamma})$. For a cooperative transmission, the average SNR of the source-to-relay or the relay-to-destination link is typically much greater than that of the source-to-destination link, i.e., $\bar{\gamma}_{s, r} \gg \bar{\gamma}_{s, d}$ and $\bar{\gamma}_{r, d} \gg \bar{\gamma}_{s, d}$. Hence, when the source transmits, the transmitted frame has a higher probability to be correctly decoded at the relay than the destination. When the relay forwards, the bit and MAC frame error rates at the destination are reduced. Packets are successfully delivered to the destination as long as either the direct transmission or the cooperative transmission is successful. The probability of a successful packet delivery for the case of one relay is expressed as

$P_{1, s u c c}=1-P_{p}\left(\bar{\gamma}_{s, d}\right)+P_{p}\left(\bar{\gamma}_{s, d}\right)\left[1-P_{p}\left(\bar{\gamma}_{s, r}\right)\right]\left[1-P_{p}\left(\bar{\gamma}_{r, d}\right)\right]$ 
In the case of $M$ multiple relays, the probability of a successful packet delivery is upper bounded by

$$
\begin{gathered}
P_{M, s u c c} \leq 1-P_{p}\left(\bar{\gamma}_{s, d}\right)+P_{p}\left(\bar{\gamma}_{s, d}\right) \times\{1- \\
\left.\Pi_{j=1}^{M}\left[1-\left(1-P_{p}\left(\bar{\gamma}_{s, r_{j}}\right)\right)\left(1-P_{p}\left(\bar{\gamma}_{r_{j}, d}\right)\right)\right]\right\}
\end{gathered}
$$

where $r_{j}$ denotes the $j$ th relay. Based on the analysis above, one can see how the probability of successful transmission can be improved as the result of using a relay. A similar conclusion can be made about the case of $M$ relays.

\section{G. Implementation Issues}

When the source-destination channel is bad, it is possible that the destination may not even decode the source MAC address correctly, and accordingly the source may never receive the ACK or NACK message due to the incorrect address. In order to combat such issue, we propose a solution that explores the MAC address from the relay. If the BER is high in the initial packet, the destination will refer to relays to check whether the address is correct. In the data packet, the destination will compare the Src domain between the source and relay to check the correctness of source address.

In the relay forwarding scenario, it is possible that a new data packet or a new relay request is received before previous transmission is ended due to the slot-based characteristic of LA-MAC and CLA-MAC protocols. Our solution is putting the data frame into a buffer. The data frame contains information associated with the source and destination address, such that a relay can forward the data to the corresponding destination with multiple data packets in its buffer. If destination receives multiple packets that are decoded incorrectly, it will repeat the process of requesting relay transmissions and confirming the receipt of those packets until all packets in its buffer are processed.

\section{EXPERIMENTAL RESUlTS}

In this section, we perform experiments in our MANET testbed to evaluate the performance of CLA-MAC protocol. In our experiments, there four nodes, i.e., one source, one destination, and two relay candidates, are distributed in a rich scattering environment. The two relay candidates are located between the source and the destination. Consequently, the relay-destination link is typically much stronger than the source-destination link.

We utilize $2 \times 2$ antenna configuration links using space-time block codes (STBCs) of [1] for transmit and maximum ratio combining (MRC) for receive along with 16-QAM modulation and a $2.48 \mathrm{GHz}$ center frequency. A 3/4 rate convolutional coder is used for bit error correction. The high modulation scheme and code rate are used in order to increase the the probability of symbol error. The latter allows for performance profiling of our cooperative protocol. The following conditions apply to our experiments. First, we assume that the channel quality varies slowly compared to packet length transmission time alleviating the need for periodic channel measurements. Second, channel measurements are done utilizing the existing scheme of LA-MAC. Third, address field corruptions are avoided relying on channel coding.

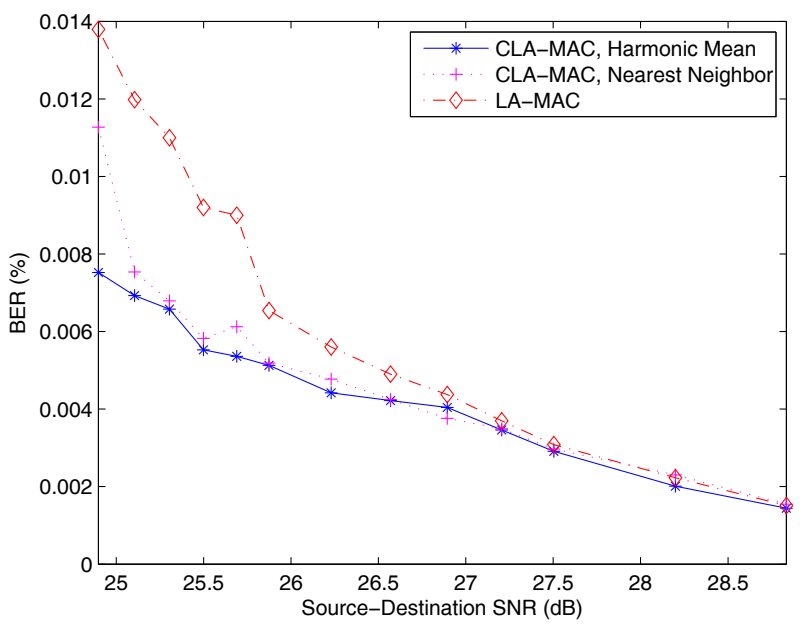

Fig. 7. A BER performance comparison of LA-MAC and CLA-MAC as a function of source-destination SNR.

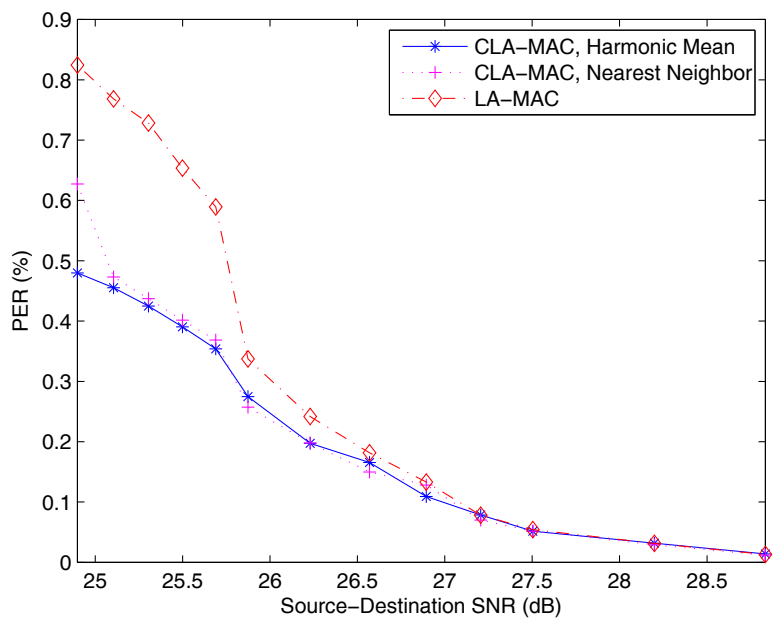

Fig. 8. A PER performance comparison of LA-MAC and CLA-MAC as a function of source-destination SNR.

Fig. 7 and Fig. 8 compare the PER and BER performance of LA-MAC and CLA-MAC using the harmonic mean and nearest neighbor policies as a function of the source-destination SNR. Both figures show that CLA-MAC using the largest harmonic mean policy enjoys an improvement of a little less than 50\% compared to LA-MAC for smaller values of SNR. The nearest neighbor policy provides only $20 \%$ improvement over LA-MAC at low SNR but as the SNR increases the difference between the policies becomes negligible. For larger SNRs, the source-destination channel becomes sufficiently strong that packet errors disappear and the relays are no longer necessary.

Fig. 9 compares the times spent using LA-MAC and CLAMAC for transmitting 500 packets correctly from the source to the destination. In the case of LA-MAC, if the destination receives an incorrect packet, it sends back a NACK and the source needs to re-send the packet. When the channel 


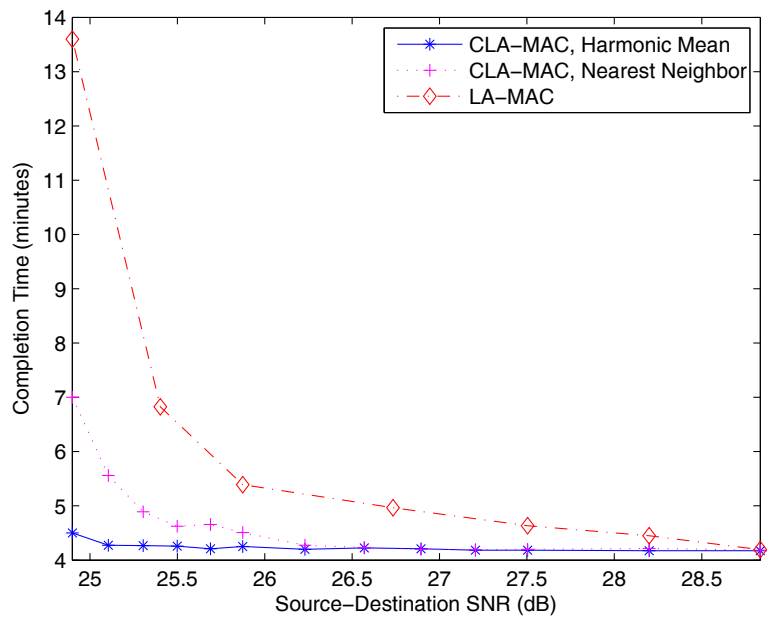

Fig. 9. A completion time comparison of LA-MAC and CLA-MAC as a function of source-destination SNR.

condition is poor, several retransmissions may be needed for delivering one packet.

In the case of CLA-MAC using the largest harmonic mean, the PER is reduced considering the fact that the relaydestination channel is usually much better than the sourcedestination channel. Therefore, the destination can receive data from a relay and there is no need for multiple retransmissions. Consequently, the completion time of the largest harmonic mean policy is much shorter than that of LA-MAC. The completion time of the nearest neighbor policy is significantly longer than the largest harmonic mean policu, but still provides $50 \%$ improvement over LA-MAC. One notices that the improvements of CLA-MAC over LA-MAC in terms of completion time are more significant for higher values of packet error rate. When the packet error rate is low, the performance of CLA-MAC is a little better than that of LAMAC.

\section{COnClusion And Future WORK}

In this paper, we designed a Cooperative MAC protocol CLA-MAC as an extension of our previously introduced LAMAC protocol. We implemented CLA-MAC protocol in our existing MANET testbed formed by a collection of USRP units running on GNU Radio. The main premise of our protocol design was that when a source-to-destination transmission fails, a relay can be selected by the destination to help retransmit the source packet using the decode-and-forward strategy. We incorporated two relay selection policies namely the largest harmonic mean and the nearest neighbor policies. Via experiments in our testbed with a small relaying topology, we confirmed that the use of CLA-MAC protocol could greatly improve the performance of LA-MAC protocol measured by factors such as packet error rate and packet delay. We are currently evaluating the performance of CLA-MAC in larger hybrid LA-MAC and CLA-MAC random topologies. We are also evaluating relaying strategies and multiple relay coordination strategies.

\section{ACKNOWLEDGMENT}

The authors would like to thank Xiaofang Liu for his early work on CLA-MAC and Weihong $\mathrm{Hu}$ for his help towards the implementation of CLA-MAC protocol based on the existing implementation of LA-MAC protocol.

\section{REFERENCES}

[1] S.M. Alamouti, "A Simple Transmitter Diversity Scheme for Wireless Communications," IEEE JSAC, November 1998.

[2] A. Azgin, Y. Altunbasak, and G. Al-Regib, "Cooperative MAC and Routing Protocols for Wireless Ad Hoc Networks," in Proc. 2005 IEEE GLOBECOM, 2005.

[3] A. Bletsas, A. Khisti, D.P. Reed, A. Lippman, "A Simple Cooperative Diversity Method Based on Network Path Selection," IEEE JSAC,March 2006.

[4] E. Blossom, "Exploring GNU radio," 2004.[Online]. Available at http://www.gnu.org/software/gnuradio.

[5] C. Chun-Ting, Y. Jun, and W. Dong, "Cooperative MAC protocol with automatic relay selection in distributed wireless networks," in Proc. IEEE PerCom Workshops, White Plains, NY, Mar. 2007, pp. 526-531.

[6] R. Dhar, G. George, A. Malani, and P. Steenkiste, "Supporting integrated MAC and PHY software development for the USRP SDR", in Proc. 1st IEEE Workshop Networking Technologies for Software Defined Radio Networks, Reston, VA, Sep. 2006.

[7] M. Ettus, "USRP product description," [Online]. Available: www.ettus.com/index.html.

[8] W. Hu, X. Li, and H. Yousefi'zadeh, "LA-MAC: A load adaptive MAC protocol for MANETs," in Proc. 2009 IEEE GLOBECOM, Honolulu, HI, Nov.- Dec. 2009

[9] A.S. Ibrahim, A.K. Sadek, W. Su, "Cooperative communications with relay-selection: When to cooperate and whom to cooperate with?", IEEE Trans. Wireless Comm., vol. 7, no. 7, pp. 2814 - 2827, Jul. 2008.

[10] H. Jiao and F.Y. Li, "Cooperative medium access control in wireless networks: the two-hop case," in Proc. IEEE WiMob 2009, Marrakech, Morocco, Oct. 2009, pp. 13-18.

[11] A. Kanzaki, T. Uemukai, T. Hara, and S. Nishio, "Dynamic TDMA slot assignment in ad hoc networks," in Proc. 17th International Conference on Advanced Information Networking and Applications, Xi'an, China, Mar. 2003, pp. 330-335.

[12] T. Korakis, Z. Tao, S.R. Singh, P. Liu, and S.S. Panwar, "Implementation of a cooperative MAC protocol: Performance and challenges in a real environment," EURASIP Journal on Wireless Communications and Networking, vol. 2009, Article ID 598140, 19 pages, 2009. doi: $10.1155 / 2009 / 598140$

[13] L. Li and H. Zhang, "A new cooperative MAC scheme for wireless ad hoc networks," in Proc. 2008 International Symposium on Information Science and Engieering, Shanghai, China, Dec. 2008, pp. 551-555.

[14] N. Li, N. Cheng, X. Xu, Y. Cai, and Y. Luo, "ARC-MAC: An automatic relay cooperative MAC protocol for wireless ad hoc networks," in Proc. 2009 International Conference on Wireless Communications and Signal Processing, Nanjing, China, Nov. 2009.

[15] X. Li, W. Hu, and H. Yousefi'zadeh, "A case study of a MIMO SDR implementation," in Proc. IEEE MILCOM, San Diego, CA, Nov. 2008.

[16] P. Liu, Z. Tao, and S. Panwar, "A cooperative MAC protocol for wireless local area networks," in Proc. IEEE ICC, Seoul, Korea, May 2005.

[17] K. Murota and K. Hirade, "GMSK modulation for digital mobile radio telephony," IEEE Trans. Commu., vol. 29, no. 7, pp. 1044-1050, Jul. 1981.

[18] M.K. Simon, M.S. Alouini, "Digital Communication over Fading Channels: A Unified Approach to Performance Analysis," John Wiley, ISBN: 0471317799, 2000

[19] L. Sun, T. Zhang, L. Lu, and H. Niu, "Cooperative communications with relay selection in wireless sensor networks," IEEE Trans. Consum. Electron., vol. 55, no. 2, pp. 513-517, May 2009.

[20] A. Sharma, V. Gelara, S.R.Singh, T. Korakis, Pei Liu, and S. Panwar, "Implementation of a cooperative MAC protocol using a software defined radio platform," in Proc. 16th IEEE Workshop on Local and Metropolitan Area Networks, Transylvania, Romania, Sept. 2008.

[21] H. Zhu, and G. Cao, "rDCF: A relay-enabled medium access control protocol for wireless ad hoc networks," IEEE Trans. Mobile Computing, vol. 5, no. 9, pp. 1201-1214, Sep. 2006.

[22] -, "Hydra: A MIMO-OFDM Multihop Testbed," http://hydra.ece.utexas.edu.

[23] -, "GNU Radio Message Blocks," http://gnuradio.org/trac/wiki/MessageBlocks. 